\title{
WHAT IF THE HEREDITARIAN HYPOTHESIS IS TRUE?
}

\author{
Linda S. Gottfredson \\ University of Delaware
}

\begin{abstract}
J. P. Rushton and A. R. Jensen (2005) review 10 bodies of evidence to support their argument that the long-standing, worldwide Black-White average differences in cognitive ability are more plausibly explained by their hereditarian $(50 \%$ genetic causation) theory than by culture-only ( $0 \%$ genetic causation) theory. This commentary evaluates the relevance of their evidence, the overall strength of their case, the implications they draw for public policy, and the suggestion by some scholars that the nation is best served by telling benevolent lies about race and intelligence.
\end{abstract}

Rushton and Jensen (2005) review the last 30 years of evidence on an important but spurned question: Is the average Black-White difference in phenotypic intelligence partly genetic in origin? Much relevant scientific evidence has accumulated since Jensen first asked the question in 1969, but openly addressing it still seems as politically unacceptable today as it was then. Taking the question seriously raises the possibility that the answer might be yes, which for some people is unthinkable. It is therefore no surprise that such research and researchers are often evaluated first against moral criteria and only secondarily, if at all, against scientific ones. My commentary therefore examines the Rushton-Jensen article against both the scientific and moral criteria typically applied to such work.

\section{The Hereditarian Hypothesis: What Is It?}

Rushton and Jensen's (2005) hereditarian hypothesis is that Black-White differences in general intelligence (IQ, or the general mental ability factor, $g$ ) are "substantially" genetic in origin, which they quantify as 50\% genetic and 50\% environmental. They specify $50 \%$ genetic because they hypothesize that race differences are simply aggregated individual differences and because researchers commonly summarize within-group IQ heritability as 50\%. Rushton and Jensen do not attempt to prove conclusively a genetic component but to show that their hypothesis is more plausible than the culture-only hypothesis long favored by social scientists, which entails $0 \%$ genetic and $100 \%$ environmental causation.

\section{Scientific Foundations of the Hereditarian Hypothesis: How Sound?}

The hereditarian hypothesis becomes scientifically plausible only after five evidentiary prerequisites have been met: IQ differences among same-race individuals represent (a) real, (b) functionally important, and (c) substantially genetic differences in general intelligence (the $g$ factor), and mean IQ differences between the races likewise reflect (d) real and (e) functionally important differences on the same $g$ factor. A century of research strongly supports all five. It has provided a

Correspondence concerning this article should be addressed to Linda S. Gottfredson, School of Education, University of Delaware, Newark, DE 19716. E-mail: gottfred@udel.edu 
vast, interlocking network of evidence that $g$ is the backbone of all broad mental abilities in all age, race, sex, and national groups studied to date; that higher levels of $g$ confer practical advantages in many realms of life; that within-group variability in phenotypic $g$ has strong genetic roots and many physiological correlates in the brain; and that between-groups differences in $g$ are large and pervasive enough to have broad social significance (e.g., see the journal Intelligence; Brody, 1992; Deary, 2000; Gottfredson, 1997; Hartigan \& Wigdor, 1989; Lubinski, 2004).

This is hardly the picture of intelligence research that the media and many social scientists paint (e.g., Fish, 2002). Both often suggest that the entire area of measurement of mental abilities, psychometrics, is fundamentally flawed and morally suspect. As Snyderman and Rothman (1988) demonstrated almost two decades ago, however, media portrayals of accepted wisdom on intelligence tend to be the opposite of what experts have actually concluded (e.g., Carroll, 1997). Thus, despite public lore to the contrary, there is already a deep and vast nomological network of evidence that can be called $g$ theory.

\section{Rushton and Jensen's 10 Bodies of Evidence: How Pertinent? How Complete?}

The most general difference between $g$ theory and culture-only theory is that the former sees both individual and group differences in $g$ as embedded substantially in biology, whereas the latter theory looks only to culture, at least when it involves race.

\section{Contrasting Predictions}

First, although both theories predict ubiquitous race differences in observed abilities, $g$ theory predicts that the gaps between any two particular races will be similar over time and place regardless of cultural circumstances (unless frequency of interbreeding changes markedly). Culture-only theory predicts that the gaps will expand or contract depending on similarity in cultural environments, regardless of genetic heritage. The uniformity of the IQ gaps between African Blacks, American Blacks, Whites, and East Asians over time and place (Rushton \& Jensen, 2005, Section 3) and the parallel ordering of race differences on simple reaction/inspection time tests in the United States and elsewhere (Section 4) are both consistent with $g$ theory. Contradicting culture-only theory, the IQ gaps fail to shift in tandem with cultural variation. Both theories can explain the BlackWhite IQ gaps seen in studies of transracial adoption (Section 7) and racial admixture (Section 8). However, the above-average mean IQ of even severely malnourished East Asian infants adopted into White European homes is more consistent with those infants having a genetic than a cultural advantage over their White European peers.

Second, unlike culture-only theory, $g$ theory predicts that IQ differences will correlate with variation in "hardwired" aspects of brain structure and function. Therefore, only $g$ theory can account for the nexus of correlations among the following outcomes: the $g$ loadedness of IQ and reaction time tests (their ability to measure $g$ ); the tests' heritability and susceptibility to inbreeding depression; Black-White-East Asian mean differences in performance on them; and the 
correlations of various physiological traits (brain size, evoked potentials in the brain, brain $\mathrm{pH}$ levels, and brain glucose metabolism) with IQ and reaction time (Sections 4 and 6).

Third, the two theories predict different degrees of change in individuals' IQs when their socioeducational environments change substantially: $g$ theory predicts little or no lasting change, but culture-only theory predicts relative responsiveness. Jensen's 1969 conclusion about the failure of socioeducational interventions to raise low IQs substantially and permanently still stands (Section 12). Natural variation in environments likewise fails to alter the common developmental processes by which abilities are assembled in different races. This commonality in cognitive architecture is indicated, for instance, by cross-race identity of $g$ factors and input-output achievement covariance matrices (Section 5). This commonality also contradicts predictions that different cultures create different intelligences.

\section{Additional Evidence}

Rushton and Jensen (2005) do not discuss one body of evidence that many social scientists believe undermines the hereditarian hypothesis: a narrowing of Black-White gaps in standardized reading achievement on the National Assessment of Educational Progress (NAEP), which critics see as a narrowing (if not the irrelevance) of the IQ gap (e.g., chapters in Jencks \& Phillips, 1998). Recent analyses (Gottfredson, in press) show the critics to be mistaken.

First, nationally representative data on racial and ethnic IQ differences during the 20th century provide no evidence that the IQ gap has narrowed. Standardized effect sizes were $1.0 \pm 0.2$ for both children and adults and for all ages and decades, averaging 1.02 across 20 samples.

Second, Black-White achievement gaps in the 1971-1999 NAEP Trend Series were no larger or smaller than $g$ theory would predict. The maximum expected is 1.20 standard deviation (the size of the Black-White $g$ gap itself), and the minimum is $0.80 \pm 0.04$ standard deviation $(1.20$ multiplied by the IQachievement correlations in core subjects). NAEP gaps narrowed from 1.07 standard deviation in the 1970s to 0.89 in the 1990s when averaged over all three subjects and ages. Degree of narrowing stalled by the mid-1980s and differed by subject: $25 \%$ in reading $(1.06-0.79), 20 \%$ in math $(1.07-0.87)$, and $15 \%$ in science (1.22-1.04). As of 1999, all gaps for 9-, 13-, and 17-year-old students were still near or above the minimum expected (reading-0.80, 0.73, 0.73; math-0.82, 0.93, 1.06; and science-0.97, 1.06, 1.07).

\section{The Totality of Available Evidence: How Compelling?}

Which theory explains the totality of evidence more consistently and coherently?

\section{Replication}

The following major facts from Rushton and Jensen's (2005) Sections 3-6 and 10-12 have been replicated many times, and all with independent sources of data. All are consistent with hereditarian theory but contradict culture-only theory: 
- Worldwide Black-White-East Asian differences in IQ (Section 3), reaction time (Section 4), and brain size, with Whites having the intermediate scores (Section 6);

- An inverse correlation between the foregoing race differences in brain attributes and Black-White-East Asian differences in body maturation (Section 6);

- Small (.2) and moderate (.4) correlations of IQ, respectively, with skull size and in vivo brain volume (Section 6);

- A moderately high correlation (usually .6-.7) of different IQ subtests' $g$ loadings, not only with the magnitude of Black-White-East Asian mean differences on those subtests (Section 6) but also with measures of those subtests' rootedness in biological and genetic processes (e.g., heritability; Section 4);

- The rising heritability of IQ with age (within races) and the virtual disappearance by adolescence of any shared environmental effects on IQ (e.g., parental income, education, child-rearing practices; Section 5);

- Worldwide Black-White-East Asian mean differences in a large suite of biological variables (e.g., twinning, gestation time, sex ratio at birth) and social variables (e.g., law abidingness, marital stability), with the three races always in the same rank order (Section 10);

- A genetic divergence (quantitative, not qualitative) of world population (i.e., racial) groups during evolution (Section 11); and

- Evidence contradicting the culture-only theory's prediction that group differences in cognitive ability should, in essence, track group differences in identifiable cultural practices and socioeconomic advantage (Section 12).

The threads of supporting evidence in Sections 5 (race-common mental architecture) and 9 (regression to the mean) tend to be less well replicated. The most direct individual tests of genetic versus environmental effects on mental ability-transracial adoption (Section 7), racial admixture (Section 8), and behavior genetic modeling of mean group differences (Section 5) - have either been uncommon or fraught with ambiguity. They clearly need to be replicated, as Rushton and Jensen (2005) suggest. Being the most direct tests of the hereditarian hypothesis, however, they are also the most politically sensitive to conduct and thus the least likely to be replicated. The more anomalous findings either require replication (e.g., training helped narrow Black African-White gaps on the Raven Matrices in some South African samples) or constitute a paradox for both theories (the Flynn effect).

\section{Consilience}

The $g$-based hereditarian theory connects $g$-related phenomena at the genetic, physiological, psychometric, and socioeconomic levels to form a coherent pattern that yields novel predictions subsequently confirmed; it is consilient. In contrast, culture-only theory has become increasingly tattered over time, patched over by disconnected ad hoc speculation.

The g-based hereditarian theory. Beginning at the psychometric level, $g$ theory has successfully predicted not only when Black-White IQ differences will remain the same in magnitude but also when they will differ markedly. First the predicted uniformity: Black-White differences are essentially the same in the West (about 1 standard deviation) across decade, age, and country, and they are 
not substantially or permanently changed by interventions intended to do so (the point of Jensen's 1969 article). This uniformity of gaps extends to three-way comparisons among Blacks, Whites, and East Asians, with East Asians outscoring Whites. Additionally, there is growing evidence for a four-way contrast, with a 1-standard deviation IQ difference-85 versus 70 -always favoring Western Blacks (who average around 20\% White admixture) over Black Africans. Regarding differences in gaps for a given race, $g$ theory successfully predicts that gaps are successively larger on more $g$-loaded tests and among children in higher social classes (in which there is more regression to the mean). The gaps thus contract and expand according to shifts in - not culture- but the cognitive demands of the tasks and individuals' genetic relatedness.

Next, this systematic patterning of Black-White-East Asian differences in performance can be traced downward from complex IQ tests, to quite elementary cognitive tasks, then to biological processes. So far, the three-way race pattern for $\mathrm{IQ} / g$ differences has been replicated with reaction/inspection time and brain size, both of which are highly heritable and correlated with $g$, as well as with a large collection of purely physical attributes (e.g., twinning). The $g$ factor is highly heritable within races and also has replicated metabolic, electrical, and structural correlates in the brain, most of them also known to be heritable (these studies are mostly with Whites).

Although Rushton and Jensen (2005) do not discuss the fact, the nexus of results for $g$ also extends outward into the social realm. For instance, the $g$ factor (indeed, the entire hierarchical structure of mental abilities; Gottfredson, in press) is the same in all races at all ages yet studied. The most $g$-loaded tests predict school and job performance best, and they predict performance equally well for Blacks and Whites in both the United States and South Africa. These findings have been replicated, but in fewer studies, for other racial and ethnic groups. The $g$ nexus goes full circle, from the social back to the genetic, because major life outcomes such as level of earnings, occupation, and education are also moderately heritable (respectively, about $40 \%-50 \%, 50 \%$, and $60 \%-70 \%$ ), with half to two thirds of their heritability being joint with $g$ (see Gottfredson, 2002, for a review; studies limited so far to European Whites).

The culture-only theory. One might be able to interpret many of the individual threads of evidence differently, but it is not clear how culture-only theory could coherently reinterpret the entire interconnected web of evidence. In fact, culture-only theory is notable for retreating from its previous failed explanations into ever-less plausible ones. For example, an early claim, plausible at the time, was that Blacks' mental abilities are underestimated because mental tests are biased against them. Research disconfirmed that claim decades ago. Although some culture-only theorists have never relinquished that belief, others began to press more vigorously the claim that any confirmed cognitive deficits among Blacks result from Blacks having suffered more than Whites from deleterious, IQ-depressing cultural conditions.

However, no such factors have been identified in genetically sensitive research. Virtually all social science claims that parental rearing and socioeconomic resources influence IQ rest on studies that confound genetic and nongenetic influences (Scarr, 1997). In fact, behavior genetic research suggests that relatively little, if any, of the Western Black-White difference in mature IQ could be due to 
the shared family factors that the culture-only theory has long presumed important (e.g., poverty, parents' education). In studies that include a broad range of family environments in Western nations, variation in such shared family factors does not create permanent within-race differences in IQ. This does not rule out the possibility that extraordinarily deleterious shared family environments permanently depress IQ, but relatively few children of any race in the West experience such extremes. As the studies of malnourished East Asian adoptees illustrate, extreme deprivation of the sort that humans have always had to contend with (e.g., starvation, infectious disease) seldom permanently impairs cognitive ability to any substantial degree once conditions are rectified.

The failure of socioeconomic resources and parenting behavior to have the influence long claimed for them led culture-only theorists to begin stressing more subtle and more race-specific psychological factors as the root cause of group differences in cognitive performance. Examples include racism-depressed motivation, racial stress, race-based performance anxiety ("stereotype threat"), and low self-esteem. All are generally posited to result in some manner from White racism and to disadvantage Blacks at all socioeconomic levels. However, there is no evidence that any of the factors causes either short- or long-term declines in actual cognitive ability. Not all of them (e.g., self-esteem) are lower for Blacks, and none can begin to explain the large array of relevant nonpsychological facts, including why the races also differ in brain size and speed (in milliseconds) of performing exceedingly simple cognitive tasks, such as recognizing which of several buttons on a console has been illuminated (a reaction time task). Because the American Black-White IQ gap has not narrowed since it was first measured in the early 1900s, the psychic injury must also be just as deleterious now as it was during that earlier, more hostile era for Blacks. This seems implausible. Thus, while the proposed psychic insults may temporarily mend some rips in the cultureonly theory, they would seem to hold even less promise than the failed socioeconomic ones for explaining the long-standing, worldwide pattern of racial IQ differences and their links to the biological correlates of $g$. The newly popular assertion that races "don't exist" is a straw man (no one believes that racial groups are biologically distinct entities), which does nothing to nullify the evidence it would have us ignore.

In summary, Rushton and Jensen (2005) have presented a compelling case that their $50 \%-50 \%$ hereditarian hypothesis is more plausible than the cultureonly hypothesis. In fact, the evidence is so consistent and so quantitatively uniform that the truth may lie closer to $70 \%-80 \%$ genetic, which is the withinrace heritability for adults in the West. The case for culture-only theory is so weak by comparison-so degenerated - that the burden of proof now shifts to its proponents to identify and replicate even one substantial, demonstrably nongenetic influence on the Black-White mean difference in $g$. Any such demonstration must be with genetically sensitive research because most "environments" are partly genetic in origin (different genotypes create and evoke different environments for themselves and their children; Scarr, 1997).

\section{Rushton and Jensen's Policy Recommendations: Are They Warranted?}

Rushton and Jensen (2005) make no recommendations for specific policies and correctly argue that the hereditarian hypothesis implies none in particular. For 
example, proof that the Black-White IQ gap is partly genetic could, depending on one's goals, be used to justify banning all racial preferences in employment and college admissions or, from a Rawlsian perspective (that genetic advantages are undeserved and unfair), require substantial and permanent racial preferences.

As Rushton and Jensen (2005) suggest, $g$ theory can predict fairly accurately how large the racial disparities in achievement will be in different settings, depending on their demands for $g$ and the IQ distributions of the groups involved. It can also provide the menu of tradeoffs between racial parity and aggregate levels of performance under different scenarios for selecting individuals into those settings, and also predict the likely pattern of effects and side effects, by race, of different interventions in education and training (e.g., Sackett, Schmitt, Ellingson, $\&$ Kabin, 2001). In short, $g$ theory can detail the challenge before us, and the likely costs and benefits of opting for different goals or means of achieving them.

Currently, racial parity in outcomes is often treated as the ultimate standard for fairness and lack of parity as a measure of White racism. For instance, disparate impact in hiring is prima facie evidence of illegal discrimination in the United States, with employers, if sued, then needing to prove themselves innocent. By undermining culture-only explanations of racial inequality, the "provisional truth" of Rushton and Jensen's (2005) hereditarian hypothesis thereby undermines the moral legitimacy of all rationales for racial equalization that posit White misbehavior as its cause. That it might persuade the public to temper or abandon its efforts to close all racial gaps in success and well-being is surely what inflames critics most.

Rushton and Jensen (2005) themselves acknowledge that open discussion of genotypic ability differences between the races might harm race relations. Their most vocal critics predict far worse. Widespread acceptance of the hereditarian hypothesis would, they say, put us on the slippery slope to racial oppression or genocide. They do not explain how this would happen but usually imply that because the Nazis were hereditarians, hereditarians must be Nazis at heart. But we can no more presume this than that IQ-environmentalists are Communists because the Communists were IQ-environmentalists. One might note, in addition, that regimes with environmentalist ideologies (Stalin and Pol Pot) exterminated as many of their citizens as did the Nazis, and virtually all the victim groups of genocide in the 20th century had relatively high average levels of achievement (e.g., German Jews, educated Cambodians, Russian Kulaks, Armenians in Turkey, Ibos in Nigeria). The critics' predictions of mass moral madness, like their frequent demonization of scientists who report unwelcome racial differences, seem mostly an attempt to stifle reasoned discussion.

But might society be better off not knowing that races differ in $g$, whether genetic or not? As Glazer (1994, p. 16) asked, "For this kind of truth, ... what good will come of it?" Summing up his argument against candor, he stated:

Our society, our polity, our elites, according to Herrnstein and Murray, live with an untruth: that there is no good reason for this [racial] inequality, and therefore society is at fault and we must try harder. I ask myself whether the untruth is not better for American society than the truth. (Glazer, 1994, p. 16)

But we must also ask, What harm might the untruth cause? Should we really presume that denying the existence of average racial differences in $g$ has only 
benefits and the truth only costs? Lying about the enduring Black-White difference in phenotypic $g$ would seem to be both futile and harmful in the long run. It is futile because the truth-and attempts to suppress it—will become increasingly obvious to the average person. Phenotypic differences in cognitive ability have relentless real-world effects that are neither ameliorated nor hidden by claims to the contrary. They also have more obvious effects in more cognitively demanding settings, such as high-level jobs and educational programs, and when entry standards differ by race.

Lying about race differences in achievement is harmful because it foments mutual recrimination. Because the untruth insists that differences cannot be natural, they must be artificial, manmade, manufactured. Someone must be at fault. Someone must be refusing to do the right thing. It therefore sustains unwarranted, divisive, and ever-escalating mutual accusations of moral culpability, such as Whites are racist and Blacks are lazy.

\section{Does the Hereditarian Hypothesis Leave Us Without Hope?}

Given what we know about $g$ 's nature and practical importance, Black-White genetic differences in $g$ render the goal of full parity in either IQ or achievement unrealistic. This does not rule out the possibility of reducing the disparities, especially in achievement, nor does it provide any reason to "give up" on anyone or conclude that some people "can't learn." In fact, rather than seeking racial parity in all outcomes, we might do better by helping lower-IQ individuals of all races. The weaker learning and problem-solving abilities of people in the lower part of the IQ distribution make their daily lives much more difficult and hazardous.

We might especially target individuals below IQ 80 for special support, intellectual as well as material. This is the cognitive ability ("trainability") level below which federal law prohibits induction into the American military and below which no civilian jobs in the United States routinely recruit their workers. It includes about $10 \%$ of Whites and a third of Blacks in the United States and the segment of both groups most at risk for multiple health and social problems, regardless of family background and material resources (Gottfredson, 1997, 2002). Moreover, the risks that lower-IQ people face in relation to more able individuals have been growing as the complexity of work, health care, and daily life has increased. The $g$ theory suggests that their relative risk might be lowered if (a) education and training were better targeted to their learning needs (instruction is more narrowly focused, nontheoretical, concrete, hands-on, repetitive, personalized, and requiring no inferences); (b) they were provided more assistance and direct instruction in matters of daily well-being that we expect most people acquire on their own (e.g., learning how best to avoid various kinds of illness and injury); and (c) health care providers, social service agencies, and other institutions removed some of the unnecessary complexity (e.g., inadequate or overly complex labeling, instructions, and forms) that often impedes full and effective use of services, medical regimens, and preventive care by the less able. Less favorable genes for $g$ impose constraints on individuals and their helpers, but they certainly do not prevent us from improving lives in crucial ways. 


\section{References}

Brody, N. (1992). Intelligence (2nd ed.). San Diego, CA: Academic Press.

Carroll, J. B. (1997). Psychometrics, intelligence, and public perception. Intelligence, 24(1), 25-52.

Deary, I. J. (2000). Looking down on human intelligence: From psychometrics to the brain. Oxford, England: Oxford University Press.

Fish, J. M. (Ed.). (2002). Race and intelligence: Separating science from myth. Mahwah, NJ: Erlbaum.

Glazer, N. (1994, October 31). The lying game. The New Republic, 15-16.

Gottfredson, L. S. (Ed.). (1997). Intelligence and social policy [Special issue]. Intelligence, 24(1).

Gottfredson, L. S. (2002). g: Highly general and highly practical. In R. J. Sternberg \& E. L. Grigorenko (Eds.), The general factor of intelligence: How general is it? (pp. 331-380). Mahwah, NJ: Erlbaum.

Gottfredson, L. S. (in press). Implications of cognitive differences for schooling within diverse societies. In C. L. Frisby \& C. R. Reynolds (Eds.), Comprehensive handbook of multicultural school psychology. New York: Wiley.

Hartigan, J. A., \& Wigdor, A. K. (Eds.). (1989). Fairness in employment testing: Validity generalization, minority issues, and the General Aptitude Test Battery. Washington, DC: National Academy Press.

Jencks, C., \& Phillips, M. (Eds.). (1998). The Black-White test score gap. Washington, DC: Brookings Institution Press.

Jensen, A. R. (1969). How much can we boost IQ and scholastic achievement? Harvard Educational Review, 39, 1-123.

Lubinski, D. (Ed.). (2004). Cognitive abilities: 100 years after Spearman's (1904) “'General Intelligence,' Objectively Determined and Measured" [Special section]. Journal of Personality and Social Psychology, 86, 96-199.

Rushton, J. P., \& Jensen, A. R. (2005). Thirty years of research on race differences in cognitive ability. Psychology, Public Policy, and Law, 11, 235-294.

Sackett, P. R., Schmitt, N., Ellingson, J. E., \& Kabin, M. B. (2001). High-stakes testing in employment, credentialing, and higher education: Prospects in a post-affirmativeaction world. American Psychologist, 56, 302-318.

Scarr, S. (1997). Behavior-genetic and socialization theories of intelligence: Truce and reconciliation. In R. J. Sternberg \& E. L. Grigorenko (Eds.), Intelligence, heredity, and environment (pp. 3-41). New York: Cambridge University Press.

Snyderman, M., \& Rothman, S. (1988). The IQ controversy, the media and public policy. New Brunswick, NJ: Transaction Press. 